\title{
A Cervical Mass in an HIV-Positive Patient
}

\author{
Seyed Alireza Mousavi, Ali Asadollahi-Amin \\ Department of Infectious Disease, Imam Khomeini Hospital, Tehran University of Medical Sciences, Tehran, Iran
}

\begin{abstract}
We describe an HIV-infected man with a left side cervical swelling which finally diagnosed with tuberculosis.In contrast to HIV-negative persons, nearly all tuberculous lymphadenitis (scrofula), the most kind of extrapulmonary tuberculosis, in patients with AIDS is multifocal. Constitutional symptoms such as fever, weight loss, and evidence of the lung involvement (parenchyma, nodes, or pleura) or elsewhere are seen in this setting. Positive findings on aspiration material are more frequently seen than HIV-negative population. J Microbiol Infect Dis 2018; 8(1):41-42
\end{abstract}

Keywords: Tuberculosis, HIV, cervical mass, clinical image

\section{CASE SUMMARY}

A 39-year-old man presented with a left side cervical swelling within a prior week (Figure 1). $\mathrm{He}$ was diagnosed with HIV, infections past eight years. However, he did not enter care at all. He had a history of intravenous cocaine use ten years ago; 3 years of jail; a cough with sputum, and weight loss in past month. He did not remind history of tuberculosis in his family. On examination, a non-tender firm erythematous mass measuring $5 \times 2 \mathrm{~cm}$ in the both left anterior and posterior triangle; two small, non-tender, firm lymph nodes in the right posterior cervical chain; and fine crackles in the base of right lung were noted. Other evaluations were unremarkable.

Laboratory assessments showed: WBC $8500 / \mathrm{mm}^{3}, \mathrm{Hb} 8.3 \mathrm{mg} / \mathrm{dl}$, PLT $275,000 / \mathrm{mm}^{3}$, AST 53 IU/I, ALT 37 IU/I, ALP 630 IU/I, ESR 135 $\mathrm{mm} / \mathrm{h}, \quad$ CD4 10 cells $/ \mathrm{mm}^{3}$. Lung computed tomography (CT) scan revealed a patchy consolidation in the right lung and diffuse centrilobular nodules in both lungs consistent with tuberculosis (Figure 2).

Multiple mediastinal lymphadenopathies measuring $62 \times 30 \mathrm{~mm}$ in superior mediastinum, reactive lymphadeno-pathies in both sides of axilla regions, and no pericardial or pleural effusions also were noted on CT scan.

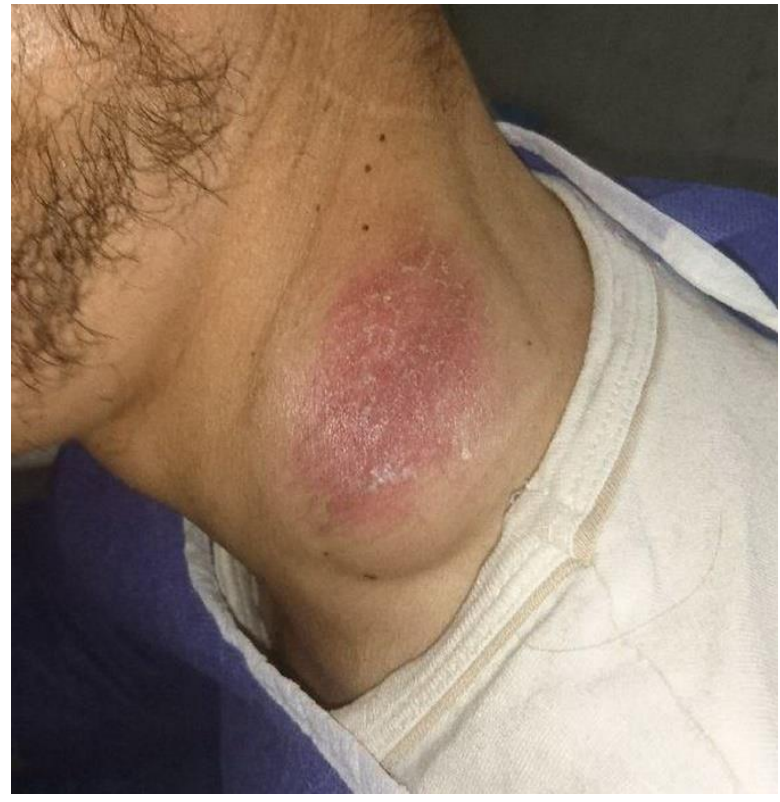

Figure 1. Figure 1. An erythematous mass on left side of the neck in a 39-year-old HIV-positive patient.

Lymph nodes with liquefaction necrosis, and collection in the lesion, as well as diffuse cervical lymphadenopathy, were found on sonography. Cultures of fluid collection and sputum were positive for Mycobacterium tuberculosis. Appropriate treatments were initiated, and the patient recovered without any complications.

Four-drug regimens (isoniazid, rifampin, pyrazinamide, and ethambutol) for 2 months, followed by isoniazid and rifampin for 4 months, 
as well as complete excision of undrained nodes, is recommended [1].

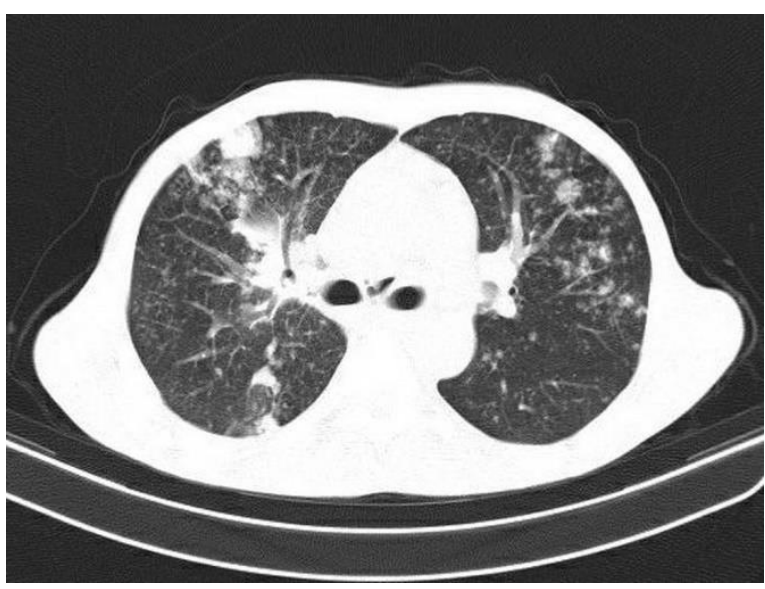

Figure 2. A patchy consolidation in right lung and diffuse centrilobular nodules (tree-in-bud opacities) in both lungs.

\section{ACKNOWLEDGMENTS}

Conflict of interest: The authors declare no personal or financial conflict of interest.

Financial Disclosure: No financial support was received

\section{REFERENCES}

1. Daniel W. Fitzgerald, Timothy R. Sterling, Haas DW. Mycobacterium tuberculosis in Mandell, Douglas, and Bennett's Principles and Practice of Infectious Diseases, 8th ed. Copyright 2015 by Saunders, an imprint of Elsevier Inc; 251: 2787-2818. 